\title{
Social media language of healthcare super-utilizers
}

\author{
Sharath Chandra Guntuku (DD ${ }^{1,2,3 凶}$, Elissa V. Klinger ${ }^{1,4}$, Haley J. McCalpin ${ }^{1,4}$, Lyle H. Ungar ${ }^{1,2,5}$, David A. Asch ${ }^{3,4,5,6}$ and \\ Raina M. Merchant ${ }^{1,3,4}$
}

An understanding of healthcare super-utilizers' online behaviors could better identify experiences to inform interventions. In this retrospective case-control study, we analyzed patients' social media posts to better understand their day-to-day behaviors and emotions expressed online. Patients included those receiving care in an urban academic emergency department who consented to share access to their historical Facebook posts and electronic health records. Super-utilizers were defined as patients with more than six visits to the Emergency Department (ED) in a year. We compared posts by super-utilizers with a matched group using propensity scoring based on age, gender and Charlson comorbidity index. Super-utilizers were more likely to post about confusion and negativity $(\mathrm{D}=.65,95 \% \mathrm{Cl}-[.38, .95])$, self-reflection $(\mathrm{D}=.63[.35, .91])$, avoidance $(\mathrm{D}=.62[.34, .90])$, swearing $(\mathrm{D}=.52[.24, .79])$, sleep $(D=.60[.32, .88])$, seeking help and attention $(D=.61[.33, .89])$, psychosomatic symptoms, $(D=.49[.22, .77])$, self-agency $(D=.56[.29, .85])$, anger $(D=.51,[.24, .79])$, stress $(D=.46,[.19, .73])$, and lonely expressions $(D=.44,[.17, .71])$. Insights from this study can potentially supplement offline community care services with online social support interventions considering the high engagement of super-utilizers on social media.

npj Digital Medicine (2021)4:55; https://doi.org/10.1038/s41746-021-00419-2

\section{INTRODUCTION}

Super-utilizers are patients with frequent acute (i.e., emergency department) healthcare encounters, often because of complex physical, behavioral, and social needs. Parameters of defining healthcare super-utilizers vary across the literature, but the Center for Medicare and Medicaid Services (CMS) defines super-utilizers as "patients who accumulate large numbers of emergency department (ED) visits and hospital admissions, which might have been prevented by relatively inexpensive early interventions and primary care". ${ }^{1}$ Super-utilization can result from or contribute to uncoordinated care and avoidable utilization of inpatient and emergency room services, and poorer health outcomes overall ${ }^{2}$. The cost implications of super-utilization are significant, with a 2012-2013 analysis $^{3}$ in the United States (US) demonstrating that $50 \%$ of healthcare expenditures were attributed to just $5 \%$ of the population. Further costs are incurred with the common comorbidities seen among super-utilizers, including mental health and substance use diagnoses ${ }^{4}$.

Conventional approaches to managing this population rely on comprehensive care coordination, community-based care, and greater attention to complex social needs. However, combined approaches are difficult to implement and expensive ${ }^{4}$ because they are applied broadly, in contrast to narrower interventions targeting specific patient populations and their socio-contextual and behavioral needs. One example of this comprehensive approach that received national attention was carried out by the Camden Coalition of Healthcare Providers. Results of their randomized controlled trial revealed that the intervention-which involved care coordination among nurses, social workers, and community health workers-did not have any significant effect on reducing readmission rates 180 days following hospital discharge ${ }^{5}$. Patient navigator interventions targeting super-utilizers have shown mixed or moderate success in reducing hospital utilization ${ }^{6,7}$.
The Camden Coalition and others rely on the incorporation of social determinants of health into their proposed interventions ${ }^{8}$. However, as lovan et al. (2019) found in a comprehensive review of super-utilizer interventions, most interventions target the downstream determinants surrounding patients' material conditions (e.g. access to housing, food, and transportation), with only a select few offering interventions targeting the more fundamental social determinants of health with referrals to education, job opportunities, and vocational training ${ }^{9-11}$. Tackling these fundamental determinants requires a more targeted approach that can be tailored to the individual and engages the patient, their families, and caregivers beyond conventional healthcare visits.

Digital engagement is increasingly sought to effectively 'hover' over patients outside of their traditional healthcare encounters $^{12}$. Smartphones, wearable devices, and social media may offer opportunities to engage patients, including superutilizers, in care that meets them where they are-both physically and metaphorically-while simultaneously offering a more cost-effective and proactive approach to solving for patient needs. Facebook-used by $69 \%$ of adults in the US, of whom $74 \%$ report using it at least once a day ${ }^{13}$ - provides both metadata and user-generated content, and thus offers a unique window into the lives of patients, and may reveal potential opportunities for interventions.

In this study, we sought to understand the online activity of consenting healthcare super-utilizers, specifically their day-to-day lifestyle, behaviors, and emotions by comparing their entire timeline of Facebook posts (quantified by open-vocabulary topics, dictionary-based psycholinguistic categories, linguistic markers of anger, stress, and loneliness expressions) with those of a matched group using propensity scoring based on Charlson comorbidity index, gender, and age.

\footnotetext{
${ }^{1}$ Penn Medicine Center for Digital Health, Philadelphia, PA, USA. ${ }^{2}$ Department of Computer and Information Science, University of Pennsylvania, Philadelphia, PA, USA. ${ }^{3}$ Perelman School of Medicine, University of Pennsylvania, Philadelphia, PA, USA. ${ }^{4}$ Penn Medicine Center for Health Care Innovation, Philadelphia, PA, USA. ${ }^{5}$ The Wharton School, University of Pennsylvania, Philadelphia, PA, USA. ${ }^{6}$ Cpl Michael J Crescenz VA Medical Center, Philadelphia, PA, USA. ${ }^{凶}$ email: sharathg@upenn.edu
} 
Table 1. Demographic characteristics and diagnoses distribution of healthcare super-utilizers and the control group.

\begin{tabular}{|c|c|c|}
\hline & Super-utilizers & Control \\
\hline Total participants & 109 & 109 \\
\hline \multicolumn{3}{|l|}{ Race } \\
\hline African American & $98(89 \%)$ & $83(76 \%)$ \\
\hline White & $11(11 \%)$ & $23(21 \%)$ \\
\hline Other & 0 & $3(3 \%)$ \\
\hline Females & 90 (83\%) & 94 (86\%) \\
\hline Age range (yrs) and IQR & {$[20,58] ; 8$} & {$[20,84] ; 34$} \\
\hline Age median (yrs) & 28 & 31 \\
\hline $\begin{array}{l}\text { Charlson Comorbidity Score range } \\
\text { and IQR }\end{array}$ & {$[-16,57] ; 7$} & {$[-9,28] ; 5$} \\
\hline \multicolumn{3}{|l|}{ Distribution of top diagnoses } \\
\hline Injury and poisoning & 92 (84\%) & $60(55 \%)$ \\
\hline Respiratory symptoms & 81 (74\%) & $51(46 \%)$ \\
\hline Skin disorders & $74(67 \%)$ & $45(41 \%)$ \\
\hline Anxiety & 38 (34\%) & $15(13 \%)$ \\
\hline Depression & $43(39 \%)$ & $23(21 \%)$ \\
\hline Drug abuse & 21 (19\%) & $6(5 \%)$ \\
\hline
\end{tabular}

\section{RESULTS}

\section{Characteristics of study subjects}

We defined super-utilizers a priori as any patient who had six or more ED encounters within a 12-month time period within our urban health system ${ }^{14}$. Out of 1830 who shared Facebook data and electronic health records as part of the Social Mediome study cohort $^{15}, 109$ participants met our criteria and were thus categorized as super-utilizers (median age of 28 and 83\% women) (Table 1). The control group included 109 participants (median age of 31 and $86 \%$ women). In our cohort, super-utilizers had more documented diagnoses of injury and poisoning, respiratory symptoms, skin disorders, anxiety, depression, and documented drug use when compared to the control group (Chi-squared statistic significant at $p<.001$ ), consistent with previous findings on the prevalence of comorbidities among super-utilizers ${ }^{1}$. Superutilizers also had on average two times more posts $(N=1537$ posts/user) in their social media profile compared to the control group ( $N=963$ posts/user), significant at $p<.05$ two-tailed $t$-test.

\section{Differentially expressed language features in the super- utilizer group}

Dictionary-based. Super-utilizers used more self-references, first person pronouns (Cohen's $D=.75,[0.47,1.05])$, words indicating present focus $(D=.57,[.29, .86])$, and function words such as adverbs $(\mathrm{D}=.53,[.26, .81])$ and negations $(\mathrm{D}=.49,[.22, .78])$ (Table 2$)$. They also used words indicative of cognitive processes including differentiation $(D=.47,[.2, .76])$, tentativeness $(D=.38$, $[.11, .66])$, and discrepancies $(\mathrm{D}=.37,[.1, .64])$.

Open-vocabulary. Compared with the control group, superutilizers were more likely to post about confusion and negativity ('erked', 'pissed', 'upset', 'confused', 'rite', D = .65, 95\% Cl-[.38, .95]), self-reflection ('mind', 'thinking', 'alot', 'much', 'head', D =.63 [.35, .91]), avoidance ('wanna', 'away', 'far', 'stay', 'cry', D = .62 [.34, .90]) and swearing, $\mathrm{D}=.52[.24, .79]$, sleep ('fall', 'sleep', 'asleep', 'bed', 'down', D =.60 [.32, .88]), seeking help and attention ('need', 'help', 'someone', 'please', 'come', 'save', $D=.61 \quad[.33, .89])$,
Table 2. Linguistic Enquiry Word Count (LIWC) categories significantly associated with language used in Facebook posts of healthcare superutilizers.

\begin{tabular}{|c|c|c|}
\hline Category & Cohen's D & $95 \% \mathrm{Cl}$ \\
\hline \multicolumn{3}{|l|}{ Pronouns } \\
\hline 1st person pronouns & 0.75 & {$[0.47,1.05]$} \\
\hline Impersonal pronouns & 0.41 & {$[0.14,0.68$} \\
\hline \multicolumn{3}{|l|}{ Time orientation } \\
\hline Present focus & 0.57 & {$[0.29,0.86]$} \\
\hline \multicolumn{3}{|l|}{ Perceptual processes } \\
\hline Feeling & 0.56 & {$[0.29,0.85]$} \\
\hline \multicolumn{3}{|l|}{ Bodily processes } \\
\hline Body & 0.54 & {$[0.26,0.82]$} \\
\hline Sexual & 0.49 & {$[0.22,0.77]$} \\
\hline \multicolumn{3}{|l|}{ Cognitive processes } \\
\hline Differentiation & 0.47 & {$[0.20,0.76]$} \\
\hline Tentativeness & 0.38 & {$[0.11,0.66$} \\
\hline Discrepancies & 0.37 & {$[0.1,0.64]$} \\
\hline \multicolumn{3}{|l|}{ Drives } \\
\hline Reward & 0.47 & {$[0.20,0.75]$} \\
\hline \multicolumn{3}{|l|}{ Function words } \\
\hline Adverbs & 0.53 & {$[0.26,0.81]$} \\
\hline Negations & 0.49 & {$[0.22,0.78$} \\
\hline \multicolumn{3}{|l|}{ Negative emotions } \\
\hline Swearing & 0.35 & {$[0.08,0.63]$} \\
\hline
\end{tabular}

psychosomatic symptoms ('pain', 'hurt', 'killing', 'ugh', 'feeling', $\mathrm{D}=.49[.22, .77])$, and self-agency ('make', 'sure', 'things', 'move', 'decisions', $\mathrm{D}=.56[.29, .85]$ ) (Table 3). Some of the highly correlated words are colloquial variations used on social media (e.g., 'erkerd' and 'rite').

Mental well-being. Super-utilizers were more likely to have posts containing language associated with anger $(D=.51,[.24, .79])$, stress $(D=.46,[.19, .73])$, and lonely expressions $(D=.44,[.17$, $.71])$. Language related to depression $(D=.23,[.03,0.5])$ and anxiety $(D=.20,[.06, .47])$ was only slightly elevated compared to the control group.

\section{DISCUSSION}

In this study, we identified themes and contexts associated with ED super-utilizer posts on Facebook that reflected stress, anger, avoidance, attention-seeking, self-reflection, and health symptoms. Many of the topics reflect social-contextual challenges that may be contributing to healthcare seeking behaviors. Prior work has shown that super-utilizers are more likely to have complex physical, behavioral, and social needs ${ }^{16}$. Our work demonstrates that these complex circumstances are in fact reflected in the social media behaviors of this patient population as measured through linguistic characteristics that demonstrate stress, conflict, and loneliness. Future studies could investigate the extent to which social media posting and behavior-including language, images, and 'lurking' time-accurately reflect the lived experience of patients. Any approach involving personalized interventions would require significant technical infrastructure and thorough ethical review to guard against further stigmatization of an already vulnerable population. 
Table 3. Topics (clusters of co-occurring words) significantly associated with language used in Facebook posts of healthcare super-utilizers.

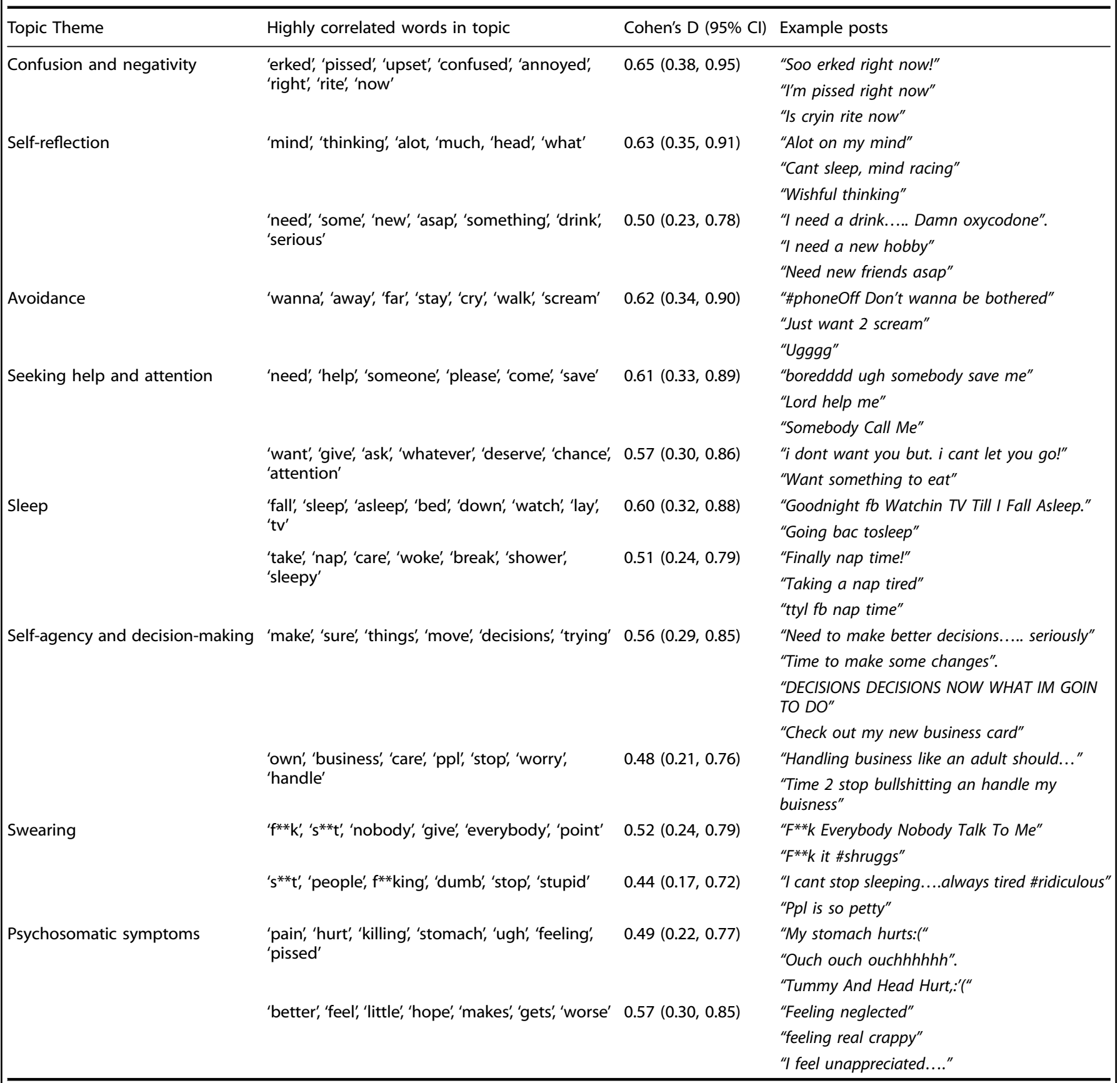

Effect size is measured using Cohen's D. Topics were categorized into themes based on a review of the posts most associated with the topic. All categories shown are significant after Benjamini-Hochberg p-correction $(p<0.01)$.

Super-utilizers tend to have more severe and uncontrolled chronic illness ${ }^{2}$; the volume of language about psychosomatic symptoms posted by super-utilizers compared with the control group in this sample supports this finding. Attention-seeking language may reflect unmet needs in the daily experiences of super-utilizers and could also be a marker of loneliness, social isolation, or underlying mental health diagnoses. The burden of mental health in populations of super-utilizers has been well documented ${ }^{2}$, so the relationship between psychiatric conditions, social vulnerability, and language on social media is plausible.

\section{Implications on intervention design}

Much of the existing literature uses payer data to identify commonalities among super-utilizer patient profiles ${ }^{4,14,17}$. Among the published interventions, these data are augmented with patient interviews and assessments to gauge access to resources (e.g., social supports, living and working circumstances, and food security), which can then inform a case management method for providing targeted support to the patient ${ }^{14,18-20}$. While our findings support the characterizations of super-utilizers published in previous literature, they also suggest a potential application in future targeted interventions. Utilizing nontraditional digital 
sources to characterize the expressions of super-utilizers may allow care teams-particularly social workers and care coordinators - to understand essential elements of a patient's daily life that may allow for a more tailored course of action to address healthcare and other needs. Such a model would require patients to share social media data with their care teams, which has several technical and ethical ramifications.

Social media analysis can potentially be used to supplement offline community care services with online social support interventions considering the high engagement of super-utilizers on social media. Engaging patients online also holds potential for increased interactive support ${ }^{21}$. While exploring digital social support groups for cancer patients, online environments were found to provide a platform for asking questions, communicating personal experiences, and sharing emotions ${ }^{22}$. In harnessing the dynamic nature of these platforms, interventions targeted to super-utilizers could respond and adapt to these highly engaged patients in an easily accessible and familiar environment. Opportunities are also growing in the development of new digital health technologies. Prior work explored super-utilizer receptivity to digital technologies for care management and outlined key takeaways from focus groups including widespread interest in digital health tools, healthcare delivery navigation challenges, and age-based digital literacy ${ }^{23}$. Our data provide further insight into super-utilizers' digital presence that could benefit future development of digital health technologies targeted to this population.

\section{Ethics and privacy}

Maintaining privacy and confidentiality are critical when looking toward healthcare applications of social media data ${ }^{24}$. Potential stigmatization of already vulnerable service users once they have been flagged as potential super-utilizers could be problematic and should be guarded against. Specific guidelines for social media health research should include strict protocols around protection measures for sensitive data and deidentification whenever possible, as well as data storage on HIPAA-compliant servers ${ }^{25}$. Such safeguards are one approach for protecting against any downstream insurance or employment consequences in the event of data breaches. Furthermore, any personalized interventions utilizing such data should place high value on maintaining patient agency and avoid any prescriptive measures based unilaterally on social media insights. Lastly, it is important to preserve trust in the relationship between provider and patient, especially among vulnerable populations. A note of caution is that introducing social media data into the patient-physician relationship can result in a patient's privacy feeling violated or influence a provider in their treatment $^{26}$.

\section{Limitations}

This study has several limitations. First, although the demographics of our sample are similar to the overall population served by the ED in urban hospitals ${ }^{15}$, our sample is not representative of the general population and is skewed towards younger African American females. Payer data revealed that super-utilizers with Medicaid coverage were older than other Medicaid patients, with an average age of 32.3 years for super-utilizers compared to 24.2 for patients with less than 6 hospital visits per year ${ }^{14}$. We prioritized matching on gender, the Charlson comorbidity score, and age (in that order). We found age and race to be significantly different across groups. In prior work, it was found that gender has the highest effect on language, but does not change a lot after 45 years, which was the reason for our characterization ${ }^{27}$. Previous literature found that super-utilizers, compared to 'low-utilizers', are more likely to be male and African American ${ }^{28}$ and Hispanic/Latino ${ }^{29}$.

Second, the EHR data for visits is obtained from one health system whereas patients might have received care from other systems not captured in our analysis. Third, though the exclusion of non-English speaking participants avoids cultural confounders considering the specific recruitment location of participants, it introduces sampling bias. Further, patients who are willing to share social media data may tend to be "over-sharers" so that the conclusions drawn may not be generalizable to all ED superutilizers, and especially because eligibility was limited to Englishspeakers and English language posts.

In summary, social media language offers a window into patients' characteristics that cannot be gleaned from their health records alone and may eventually lead to new ways to identify needs at the individual or population level. Healthcare superutilizers' social media posts reveal themes that suggest lifestyles, behaviors, and emotions that reflect negativity, conflict, sleep deprivation, and psychosomatic symptoms. While these findings need to be replicated in other studies before implementing interventions, this study is a step towards considering the inclusion of patient-generated data, with explicit consent, in understanding healthcare needs and sequelae-providing insight and a comprehensive view of the challenges these patients face beyond their medical presentation.

\section{METHODS}

\section{Study design and setting}

The study was approved by the University of Pennsylvania Institutional Review Board. Using a convenience sample framework, from March 2014 through December 2017 patients receiving care in the emergency department (ED) of an urban academic hospital system were approached about participating in a study to merge social media and Electronic Health Records (EHR) data ${ }^{15}$. All participants gave their written informed consent to use their data for this study.

\section{Selection of participants}

We retrieved Facebook status updates up to 5 years prior to the ED index visit for all participants who consented to share their Facebook posts $(N=4587)$. We did not access data from the Facebook pages of study participants' friends or from posts on the study participants' pages made by anyone other than the participant. We excluded nonEnglish posts and selected users with a minimum of 400 words, determined from prior work to be the minimum threshold for reliably predicting user traits from language ${ }^{30}$, retaining 1830 participants with Facebook data.

Extracting data from the EHR, we identified the ED visits for these participants which coincided with years when they also had Facebook data. We first identified all years (from 2009 to 2016) in which participants had six or more ED visits. For each patient, we obtained primary ICD-9 codes of every ED and inpatient visit available in the EHR. Then, we used these ICD-9 codes to obtain the diagnoses by mapping them onto the categories in Elixhauser comorbidity $\operatorname{codes}^{31}$. We used these categories to identify differences in diagnoses across super-utilizer and control groups. Further, using the same ICD-9 codes, we calculated the Charlson comorbidity index to obtain a measure of severity of disease for every patient. We characterized patients with six or more ED visits in any year from 2009 to 2016 as super-utilizers - as most of them had contiguous hospital visits in these years ${ }^{16}$. Since healthcare utilization varies based on demographics and severity of illness, we identified a propensity score matched group of control users based on the Charlson comorbidity index, gender, and age of our super-utilizer set in a retrospective case-control manner.

\section{Linguistic attributes}

We characterized posts using three sets of language features: (a) dictionary-based psycholinguistic features, (b) open-vocabulary topics ${ }^{32}$, and (c) mental well-being attributes, such as anger, anxiety, depression, stress, and lonely expressions by applying previously developed predictive models $^{33-35}$

Dictionary-based. From each post, we extracted the relative frequency of words/tokens. We removed words used by less than $1 \%$ of users. We then compared the posts of the super-utilizer and control groups against the 73 psycholinguistic categories from the Linguistic Inquiry Word Count 
$(\text { LIWC) })^{36}$. For each, we measured the proportion of tokens (including words, emoticons etc.) represented in each LIWC category.

Open-vocabulary. We also used an open-vocabulary approach. Two hundred latent Dirichlet allocation (LDA) topics (groups of co-occurring words) were generated using Facebook posts contributed by patients from a prior study ${ }^{32}$. The LDA generative model assumes that posts contain a combination of topics, and that topics are a distribution of words. Since the words in a post are known, topics, which are latent variables, can be estimated through Gibbs sampling. We use the Mallet implementation of the LDA algorithm, adjusting one parameter $(a l p h a=5)$ to favor fewer topics per post. All other parameters were kept at their default. An example of such a model is the following set of words ('tuesday', 'monday', 'wednesday',...) which clusters together days of the week by exploiting their similar distributional properties across tweets. We calculated the topic distribution of each user aggregated across all posts.

Mental well-being attributes. We used automatic text-regression methods developed in previous works to assign to each user scores on the depression $^{35}$, anxiety ${ }^{37}$, anger, stress ${ }^{34}$, and lonely expressions ${ }^{33}$.

\section{Identifying differentially expressed language features in the super-utilizer group}

Posts from the same years were used for both case and control groups2009-2016. We designed this as a person-level analyses and each individual was counted only once: 109 cases and 109 controls. All language features were extracted and compared at the individual level. Each linguistic attribute and mental well-being attribute were used as input in a logistic regression model. The models were setup to predict super-utilizers (i.e., group was the dependent variable). In accordance with conventional linguistic analysis, we used a $p$-value of $<.05$ for LIWC and mental health attributes and $p<.01$ for topics, after adjusting for multiple comparisons using Benjamini-Hochberg correction, to identify potentially meaningful associations. We calculated Cohen's D associated with the super-utilizer's group with the control group as reference, for each retained attribute ${ }^{38}$.

\section{Reporting summary}

Further information on research design is available in the Nature Research Reporting Summary linked to this article.

\section{DATA AVAILABILITY}

Deidentified data necessary to reproduce the results contained in the document are available upon request. We will not, however, share individual-level Facebook data as it contains potentially identifying information about patients enrolled in the study.

\section{CODE AVAILABILITY}

Analysis code is released as part of the Differential Language Analysis Toolkit (http:// dlatk.wwbp.org).

Received: 22 July 2020; Accepted: 16 February 2021; Published online: 25 March 2021

\section{REFERENCES}

1. Mann, C. Targeting Medicaid super-utilizers to decrease costs and improve quality. Cent. Med. Med. Serv. https://www.hhs.gov/guidance/sites/default/files/ hhs-guidance-documents/CIB-07-24-2013_12.pdf (2013).

2. $\mathrm{Ng}, \mathrm{S}$. H.-X. et al. Characterization of high healthcare utilizer groups using administrative data from an electronic medical record database. BMC Health Serv. Res. 19, 452 (2019).

3. Cohen, S. B. The concentration and persistence in the level of health expenditures over time: estimates for the US Population, 2012-2013. https://www.ncbi.nlm.nih. gov/books/NBK447174/ (2001).

4. Hasselman, D. Super-Utilizer Summit: Common Themes from Innovative Complex Care Management Programs. https://www.chcs.org/media/FINAL_Super-Utilizer_Report. pdf (2013).

5. Finkelstein, A., Zhou, A., Taubman, S. \& Doyle, J. Health care hotspotting' A randomized, controlled trial. N. Engl. J. Med. https://doi.org/10.1056/NEJMsa1906848 (2020)
6. Schickedanz, A. et al. Impact of social needs navigation on utilization among high utilizers in a large integrated health system: a Quasi-experimental Study. J. Gen. Intern. Med. https://doi.org/10.1007/s11606-019-05123-2 (2019).

7. Thompson, M. P. et al. Community navigators reduce hospital utilization in superutilizers. Am. J. Manag. Care. 24, 70 (2018).

8. Vaida, B. For super-utilizers, integrated care offers a new path. Health Aff. https:// doi.org/10.1377/hlthaff.2017.0112 (2017).

9. Bronsky, E. S. et al. CARES: a community-wide collaboration identifies superutilizers and reduces their 9-1-1 call, Emergency Department, and Hospital Visit Rates. Prehospital Emerg. Care https://doi.org/10.1080/10903127.2017.1335820 (2017).

10. lovan, S., Lantz, P. M., Allan, K. \& Abir, M. Interventions to decrease use in prehospital and emergency care settings among super-Utilizers in the United States: a systematic review. Med. Care Res. Rev. https://doi.org/10.1177/1077558719845722 (2019).

11. Nossel, I. R. et al. Use of peer staff in a critical time intervention for frequent users of a psychiatric emergency room. Psychiatr. Serv. https://doi.org/10.1176/appi. ps.201500503 (2016)

12. Asch, D. A., Muller, R. W. \& Volpp, K. G. Automated Hovering in Health CareWatching Over the 5000 h. N-. Engl. J. Med. https://doi.org/10.1056/nejmp1203869 (2012).

13. Perrin, A. \& Anderson, M. Social media usage in the U.S. in 2019. Pew Res Cent https://www.pewresearch.org/fact-tank/2019/04/10/share-of-u-s-adults-usingsocial-media-including-facebook-is-mostly-unchanged-since-2018/ (2019).

14. Jiang, H. J., Weiss, A. J. \& Barrett, M. L. Characteristics of Emergency Department Visits for Super-Utilizers by Payer, 2014: Statistical Brief \#221. 2006. https://www. hcup-us.ahrq.gov/reports/statbriefs/sb221-Super-Utilizer-ED-Visits-Payer-2014. jsp.

15. Padrez, K. A. et al. Linking social media and medical record data: a study of adults presenting to an academic, urban emergency department. BMJ Qual. Saf. 25, 414-423 (2016).

16. Harris, L. J. et al. Characteristics of hospital and emergency care super-utilizers with multiple chronic conditions. J. Emerg. Med. 50, e203-e214 (2016).

17. Dang, S., Nedd, N., Aguilar, E. J. \& Roos, B. A. Differential resource utilization benefits with internet-based care coordination in elderly veterans with chronic diseases associated with high resource utilization. Telemed. J. e-Health https://doi. org/10.1089/tmj.2006.12.14 (2006).

18. Green, S. R., Singh, V. \& O'Byrne, W. Hope for New Jersey's city hospitals: the Camden Initiative. Perspect. Health Inf. Manag. (2010).

19. Shearer, A. J., Hilmes, C. L. \& Boyd, M. N. Community linkage through navigation to reduce hospital utilization among super utilizer patients: a case study. Hawaii $\lrcorner$. Med. Public Health 78, 98 (2019).

20. Okin, R. L. et al. The effects of clinical case management on hospital service use among ED frequent users. Am. J. Emerg. Med. https://doi.org/10.1053/ ajem.2000.9292 (2000).

21. Barak, A. \& Grohol, J. M. Current and future trends in internet-supported mental health interventions. J. Technol. Hum. Serv. https://doi.org/10.1080/ 15228835.2011.616939 (2011).

22. Myrick, J. G. \& Oliver, M. B. Laughing and crying: mixed emotions, compassion, and the effectiveness of a YouTube PSA about skin cancer. Health Commun https://doi.org/10.1080/10410236.2013.845729 (2015).

23. Davis, R. Digital Health Innovations for Medicaid Super-Utilizers: Consumer Feedback to Steer New Technologies. https://www.chcs.org/media/ Digital_Health_Issue_Brief_final_web1.pdf (2013).

24. Mckee, R. Ethical issues in using social media for health and health care research Health Policy 110, 298-301 (2013).

25. Benton, A., Coppersmith, G. \& Dredze, M. Ethical Research Protocols for Social Media Health Research. https://doi.org/10.18653/v1/w17-1612 (2017).

26. Denecke, K. et al. Ethical issues of social media usage in healthcare. Yearb. Med. Inform. https://doi.org/10.15265/IY-2015-001 (2015).

27. Eichstaedt, J. C. et al. Closed- and open-vocabulary approaches to text analysis: a review, quantitative comparison, and recommendations. Psychol. Methods https://psyarxiv.com/t52c6/download?format=pdf (2020).

28. Hyer, J. M. et al. Characterizing and assessing the impact of surgery on healthcare spending among Medicare enrolled preoperative super-utilizers. Ann. Surg. https://doi.org/10.1097/SLA.0000000000003426 (2019).

29. Rinehart, D. J. et al. Identifying subgroups of adult superutilizers in an urban safety-net system using latent class analysis. Med. Care https://doi.org/10.1097/ MLR.0000000000000628 (2018).

30. Jaidka, K., Guntuku, S. C., Buffone, A., Schwartz, H. A. \& Ungar, L. Facebook vs. twitter: differences in self-disclosure and trait prediction. in Proc. International AAAl Conference on Web and Social Media. (2018).

31. Elixhauser, A., Steiner, C., Harris, D. R. \& Coffey, R. M. Comorbidity measures for use with administrative data. Med. Care https://doi.org/10.1097/00005650199801000-00004 (1998) 
32. Merchant, R. M. et al. Evaluating the predictability of medical conditions from social media posts. PLOS ONE https://doi.org/10.1371/journal.pone.0215476 (2019).

33. Guntuku, S. C. et al. Studying expressions of loneliness in individuals using twitter: an observational study. BMJ Open https://doi.org/10.1136/bmjopen-2019030355 (2019).

34. Guntuku, S. C., Buffone, A., Jaidka, K., Eichstaedt, J. C. \& Ungar, L. H. Understanding and measuring psychological stress using social media. in Proc. International AAAl Conference on Web and Social Media. (2019).

35. Schwartz, H. A. et al. Towards assessing changes in degree of depression through facebook. in Proc. Workshop on Computational Linguistics and Clinical Psychology: From Linguistic Signal to Clinical Reality. (2014).

36. Pennebaker, J. W., Boyd, R. L., Jordan, K. \& Blackburn, K. The Development and Psychometric Properties of LIWC2015. http://liwc.net/LIWC2007LanguageManual. pdf (2015).

37. Guntuku, S. C., Preotiuc-Pietro, D., Eichstaedt, J. C. \& Ungar, L. H. What twitter profile and posted images reveal about depression and anxiety. in Proc. International AAAl Conference on Web and Social Media. (2019).

38. Guntuku, S. C. et al. Variability in language used on social media prior to hospital visits. Sci. Rep. https://doi.org/10.1038/s41598-020-60750-8 (2020).

\section{ACKNOWLEDGEMENTS}

This work was partly funded by Robert Wood Johnson Foundation Pioneer Award 72695 and NIH NLHBI R01HL141844. The funders did not play any role in the study design, data collection and analysis, decision to publish, or preparation of the manuscript.

\section{AUTHOR CONTRIBUTIONS}

S.C.G. and R.M.M. originated the study. S.C.G., E.V.K., L.H.U., and R.M.M. developed methods, interpreted analysis, and contributed to the writing of the article. D.A.A. and H.J.M. assisted with the interpretation of the findings and contributed to the writing of the article.

\section{COMPETING INTERESTS}

D.A. is a partner and part owner of VAL Health, and is a US government employee. The other authors have no conflicts of interest to declare.

\section{ADDITIONAL INFORMATION}

Supplementary information The online version contains supplementary material available at https://doi.org/10.1038/s41746-021-00419-2.

Correspondence and requests for materials should be addressed to S.C.G.

Reprints and permission information is available at http://www.nature.com/ reprints

Publisher's note Springer Nature remains neutral with regard to jurisdictional claims in published maps and institutional affiliations. Attribution 4.0 International License, which permits use, sharing, adaptation, distribution and reproduction in any medium or format, as long as you give appropriate credit to the original author(s) and the source, provide a link to the Creative Commons license, and indicate if changes were made. The images or other third party material in this article are included in the article's Creative Commons license, unless indicated otherwise in a credit line to the material. If material is not included in the article's Creative Commons license and your intended use is not permitted by statutory regulation or exceeds the permitted use, you will need to obtain permission directly from the copyright holder. To view a copy of this license, visit http://creativecommons. org/licenses/by/4.0/

(c) The Author(s) 2021 